\title{
A NOTE ON HOMOMORPHIC MAPPINGS OF QUASIGROUPS INTO MULTIPLICATIVE SYSTEMS
}

\author{
GRACE E. BATES AND FRED KIOKEMEISTER
}

The study of normality theories for general quasigroups and loops became productive when that study was restricted to a study of homomorphisms of quasigroups on quasigroups. ${ }^{1}$ The existence of a loop with homomorphic image which is not a quasigroup is then pertinent to this study. In this note we exhibit such a loop and show that certain properties of our example are necessary. In particular, if the homomorphic image of a quasigroup is a finite or an associative multiplicative system, this image is a quasigroup. A deeper statement is that of Theorem 4-finiteness of the kernel of a loop homomorphism into a multiplicative system is a sufficient condition for the image of this homomorphism to be a loop.

We make use of the following definitions: A multiplicative system $M$ is a nonvacuous set of elements $a, b, c, \cdots$ such that to each ordered pair of elements $a, b$, there corresponds in $M$ a uniquely defined element $a b$ called the product. If the product is defined for a (possibly vacuous) subset of the set of ordered pairs, then $M$ is called a partial multiplicative system. If $M_{1}$ and $M_{2}$ are partial multiplicative systems, $M_{1}$ is said to be imbedded in $M_{2}$ if $M_{1} \subseteq M_{2}$ and products in $M_{2}$ coincide with those in $M_{1}$ whenever they are defined in $M_{1}$. A partial multiplicative system has an identity element $e$ if the products $e a$ and $a e$ are defined for each element $a$ and $e a=a e=a$. A mapping of a multiplicative system $M$ on a multiplicative system $\bar{M}$ which preserves products is called a homomorphism of $M$. A multiplicative system $G$ in which the equations $a x=b$ and $y a=b$ have unique solutions for each pair of elements $a, b$ in $G$ is called a quasigroup. A loop is a quasigroup with identity element.

TheOREM 1. If $J$ is a partial multiplicative system, then $J$ can be imbedded in a multiplicative system $M$ which has the additional properties:

(1) If $a$ and $b$ are elements of $M$, there is at least one $x$ and at least one $y$ in $M$ such that $a x=b$ and $y a=b$.

(2) If $x \neq y$ but $a x=a y$ (or $x a=y a$ ) in $M$, then $a, x, y$, and $a x=a y$ (or $x a=y a$ ) are all in $J$. 1948.

Presented to the Society, December 31, 1948; received by the editors January 26,

${ }^{1}$ Cf. [1, p. 513]; [2, p. 450]; [3, p. 769]; [4, Theorem 10A]; and [5]. (Numbers in brackets refer to the bibliography.) 
(Note that (1) asserts that all equations are solvable in $M$, while (2) states that the solutions are unique except possibly for those equations possessing in $J$ more than one solution. Hence, in particular, if $J$ has both cancellation laws, $M$ is a quasigroup.)

We define first an elementary extension $K$ of a partial multiplicative system $J$ as follows: Let $K$ consist of all elements of $J$ together with new elements $z_{a b}, x_{a b}, y_{a b}$ defined in the following manner:

Each ordered pair of elements $a, b$ in $J$ for which $a b$ is not in $J$ gives rise to an element $z_{a b}$ in $K$, the element $z_{a b}$ being uniquely defined by the relation $z_{a b}=a b$ and the requirement that $z_{a b}=z_{c d}$ if and only if $a=c$ and $b=d$. Similarly, to each ordered pair of elements in $J$ for which there is no $x$ in $J$ satisfying $x a=b$, there corresponds an element $x_{a b}$ in $K$ for which $\left(x_{a b}\right) a=b$ is the defining relation, and to each ordered pair of elements $a, b$ in $J$ for which there is no $y$ in $J$ satisfying $a y=b$, there corresponds an element $y_{a b}$ in $K$ defined by the relation $a\left(y_{a b}\right)=b$. Again, $x_{a b}=x_{c d}$ or $y_{a b}=y_{c d}$ if and only if $a=c$ and $b=d$.

The set $K$ is a partial multiplicative system having the following properties:

(i) If $a, b$ is an ordered pair of elements in $J, a b$ is a uniquely defined element of $K$.

(ii) If $a$ and $b$ are elements of $J$, there is at least one $x$ and at least one $y$ in $K$ such that $a x=b$ and $y a=b$.

(iii) If $x \neq y$, but $a x=a y$ (or $x a=y a$ ) in $M$, then $a, x, y$, and $a x=a y$ (or $x a=y a$ ) are all in $J$.

Consider the chain of partial multiplicative systems

$$
J=J_{0} \subseteq J_{1} \subseteq J_{2} \subseteq \cdots \subseteq J_{i} \subseteq J_{i+1} \subseteq \cdots
$$

where $J_{i+1}$ is an elementary extension of $J_{i}$ for $i=1,2, \cdots$. Let

$$
M=\bigcup J_{i}
$$

be the set-theoretic sum ${ }^{2}$ of the $J_{i}$.

If $a$ and $b$ are elements of $M$, there exists an integer $k$ such that $a$ and $b$ are elements of $J_{k}$, and therefore there exists a unique element $a b$ in $J_{k+1}$. Thus to each ordered pair of elements $a, b$ in $M$ there corresponds a product $a b$ in $M$. Furthermore, if $a$ and $b$ are in $J_{k}$, then $J_{k+1}$ contains elements $x$ and $y$ such that $a x=b$ and $y a=b$.

If $x \neq y$, but $a x=a y$ in $M$, then $a, x, y$, and $a x=a y$ all lie in some $J_{k}$ and $x \neq y$ in $J_{k}$. It follows that $a, x, y$, and $a x=a y$ are elements of $J_{i}, i=k-1, k-2, \cdots, 0$; that is, these elements lie in $J$. This proves the theorem.

\footnotetext{
${ }^{2}$ Clearly $M$ is countable if $J$ is countable.
} 
COROLlary 1. If $J$ is a partial multiplicative system with identity element e, then $J$ may be imbedded in a multiplicative system $M$ with identity e, having properties (1) and (2) of the theorem.

For, in the construction of the elementary extension $K$ of $J$, we require now only the additional condition imposed on the symbols $z_{a b}, x_{a b}$, and $y_{a b}$ that they satisfy the relations $\left(z_{a b}\right) e=e\left(z_{a b}\right)=z_{a b}$, and so forth.

Suppose, now, that $J$ is commutative (that is, $a b$ is in $J$ if and only if $b a$ is in $J$, and $a b=b a$ ). Then if $a b$ is undefined in $J, b a$ is also undefined, and we may let $z_{a b}=a b=b a$ in $K$. Similarly, if there is in $J$ no solution $x$ of the equation $x a=b$, there is no solution of $a y=b$, and we may define $s_{a b}=x_{a b}=y_{a b}$ in $K$ by the relations $\left(s_{a b}\right) a=a\left(s_{a b}\right)$ $=b$. Hence we have the following corollary.

Corollary 2. If $J$ is a partial multiplicative system which is commutative, then $J$ may be imbedded in a commutative multiplicative system $M$ having properties (1) and (2) of the theorem.

We shall employ Theorem 1 in constructing an example as follows:

Let $J$ be the set consisting of the four elements $\beta_{1}, \beta_{2}, \beta_{3}, \beta_{4}$ with the following products defined:

$$
\begin{aligned}
& \beta_{1} \beta_{k}=\beta_{k} \beta_{1}=\beta_{k}, \\
& \beta_{2} \beta_{2}=\beta_{2} \beta_{4}=\beta_{4} \beta_{2}=\beta_{4} \beta_{4}=\beta_{3} .
\end{aligned} \quad k=1,2,3,4,
$$

It is to be noted that $J$ is a commutative partial multiplicative system with identity element $\beta_{1}$. The equation $\beta_{2} x=\beta_{3}$ has two distinct solutions in $J$, and thus $J$ cannot be imbedded in a quasigroup.

Let $J$ be imbedded in a system $M$, as in Theorem 1 , with elements $\beta_{k}, k=1,2,3, \cdots$. By Corollary $1, \beta_{1}$ may be taken to be the identity element of $M$. Then $M$ is a multiplicative system with the following properties:

(1) There exist positive integers $h$ and $k$ such that for each pair $\beta_{i}$ and $\beta_{m}, \beta_{i} \beta_{h}=\beta_{m}$ and $\beta_{k} \beta_{i}=\beta_{m}$.

(2) If $\beta_{i} \beta_{h}=\beta_{i} \beta_{k}$, or if $\beta_{h} \beta_{i}=\beta_{k} \beta_{i}$, where $h<k$, then $h=2, k=4$, and $i=2$ or $i=4$.

Let $A$ be a countably infinite loop ${ }^{3}$ with elements $\alpha_{1}, \alpha_{2}, \alpha_{3}, \ldots$ where $\alpha_{1}$ is the identity of $A$. We construct a system $G$ whose elements are the ordered pairs of elements $\left(\beta_{i}, \alpha_{j}\right), i, j=1,2,3, \cdots$, with $\beta_{i}$ in $M$ and $\alpha_{j}$ in $A .^{4}$ The product of two elements in $G$ is defined by:

${ }^{3} A$ may be a group.

${ }^{4}$ A similar construction has been employed by Bruck; see $[4$, p. 166]. 


$$
\left(\beta_{i}, \alpha_{j}\right)\left(\beta_{h}, \alpha_{k}\right)=\left(\beta_{i} \beta_{h}, \alpha_{n}\right)
$$

where the subscript $n$ of $\alpha_{n}$ is determined in the following way: Let $\alpha_{q}$ be the uniquely determined element $\alpha_{j} \alpha_{k}$ in $A$; then in (P)

(1) If $i=h=2$, or if $i=h=4$, let $n=2 q-1$,

(2) If $i=2, h=4$, or if $i=4, h=2$, let $n=2 q$,

(3) In all other cases, let $n=q$.

It is easily verified that $G$ is a loop with identity $\left(\beta_{1}, \alpha_{1}\right)$. The set $H$ of elements $\left(\beta_{1}, \alpha_{i}\right), i=1,2,3, \cdots$, is a loop isomorphic with $A$ under the correspondence

$$
\left(\beta_{1}, \alpha_{i}\right) \leftrightarrow \alpha_{i}, \quad i=1,2,3, \cdots .
$$

The definition of product in $G$ implies that the correspondence

$$
\left(\beta_{i}, \alpha_{j}\right) \rightarrow \beta_{i}, \quad i, j=1,2,3, \cdots,
$$

is a homomorphism of $G$ on $M$. The kernel of this homomorphism is $H$.

By Corollary 2 of Theorem 1, $M$ may be chosen to be commutative. If, furthermore, $A$ is commutative, then $G$ will have the same property. Commutativity, then, is not a sufficient condition that a quasigroup have only quasigroup images.

It is to be noted that in the example $M$ is neither finite nor associative, and $H$ is not finite. We shall show that these are necessary properties of the example.

In general, let the quasigroup $G$ be homomorphic to the multiplicative system $G^{\prime}$. If the elements of $G$ are $a, b, c, \cdots$, let $a^{\prime}, b^{\prime}, c^{\prime}, \ldots$ be their corresponding images in $G^{\prime}$. We have immediately the following lemmas:

Lemma 1. If $a^{\prime}$ and $b^{\prime}$ are any two elements of $G^{\prime}$, then $x^{\prime}$ and $y^{\prime}$ exist in $G^{\prime}$ such that $a^{\prime} x^{\prime}=b^{\prime}$ and $y^{\prime} a^{\prime}=b^{\prime}$.

LEMma 2. The system $G^{\prime}$ is a quasigroup if and only if $a^{\prime} b^{\prime}=a^{\prime} c^{\prime}$ and $b^{\prime} a^{\prime}=c^{\prime} a^{\prime}$ each implies $b^{\prime}=c^{\prime}$.

THEOREM 2. If the homomorphic image $G^{\prime}$ of a quasigroup $G$ is finite, then $G^{\prime}$ is a quasigroup. ${ }^{5}$

Let $b_{1}^{\prime}, b_{2}^{\prime}, \cdots, b_{n}^{\prime}$ be the elements of $G^{\prime}, n$ a positive integer. Then by Lemma 1 , for given $i, b_{i}^{\prime} b_{1}^{\prime}, b_{i}^{\prime} b_{2}^{\prime}, \cdots, b_{i}^{\prime} b_{n}^{\prime}$ are $n$ distinct elements of $G^{\prime}$. Right-hand cancellation is similarly established.

TheOREM 3. If the homomorphic image $G^{\prime}$ of a quasigroup $G$ is associative, then $G^{\prime}$ is a quasigroup.

${ }^{5}$ Cf. [5, Theorem 4.13]. 
A multiplicative system which satisfies Lemma 1 and is associative is known to be a group (see [7, p. 19]).

If $S$ is a subset of $G$, let $O[S]$ be the cardinal number of elements in $S$. Obviously

$$
O[S]=O[a S]=O[S a]
$$

if $a$ is any element of $G$.

We define $R(a)$ to be the set of elements $g$ in $G$ such that $g^{\prime}=a^{\prime}$ in $G^{\prime}$. Then

$$
\begin{aligned}
& R(a) b \subseteq R(a b), \quad \text { and } \\
& a R(b) \subseteq R(a b) ;
\end{aligned}
$$

for if $g=d_{1} b$ where $d_{1}^{\prime}=a^{\prime}$, then $g^{\prime}=d_{1}^{\prime} b^{\prime}=a^{\prime} b^{\prime}=(a b)^{\prime}$, and $g$ lies in $R(a b)$. The second statement follows in the same way.

Let $a$ and $b$ be any elements of $G$. There exists $x$ in $G$ such that $a=b x$. By (B), $R(b) x \subseteq R(b x)=R(a)$, and thus $O[R(b) x] \leqq O[R(a)]$. By (A), $O[R(b)]=O[R(b) x]$. It follows that $O[R(b)] \leqq O[R(a)]$. But $a$ and $b$ were any elements of $G$. We have proved the following lemma.

LEMMA 3. If $a$ and $b$ are any elements of $G$, then $O[R(a)]=O[R(b)] .{ }^{6}$

LEMMA 4. If $G^{\prime}$ is the homomorphic image of the quasigroup $G$, then $G^{\prime}$ is a quasigroup if and only if $R(a b)=a R(b)=R(a) b$.

Let $R(a b)=a R(b)=R(a) b$. If $a^{\prime} b^{\prime}=a^{\prime} c^{\prime}$, then $a c$ is an element of $R(a b)=a R(b)$, and $c$ lies in $R(b)$, that is, $c^{\prime}=b^{\prime}$. By Lemma $2, G^{\prime}$ is a quasigroup.

Conversely, let $G^{\prime}$ be a quasigroup. Let $g=x b$ be an element of $R(a b)$. Then $g^{\prime}=x^{\prime} b^{\prime}=a^{\prime} b^{\prime}$, and by Lemma 2, $x^{\prime}=a^{\prime}$. Thus $x$ lies in $R(a)$, and $g$ lies in $R(a) b$. It follows that $R(a) b \supseteq R(a b)$. By (B), however, $R(a) b \subseteq R(a b)$. Then $R(a) b=R(a b)$, and by a similar argument $a R(b)=R(a b)$.

Lemma 5. If $O[R(a)]$ is finite, $G^{\prime}$ is a quasigroup.

By (B), $R(a b) \supseteq a R(b)$. By Lemma 3 and (A), $O[R(a b)]=O[R(b)]$ $=O[a R(b)]$. Since this order is finite, $R(a b)=a R(b)$. Similarly, $R(a b)=R(a) b$, and by Lemma $4, G^{\prime}$ is a quasigroup.

THEOREM 4. If $G$ is a loop homomorphic to the multiplicative system $G^{\prime}$, and if the kernel of the homomorphism is finite, then $G^{\prime}$ is a loop.

If $e$ is the identity of $G$, then $R(e)$ is the kernel of the homomorphism. The theorem follows by Lemma 5 .

\footnotetext{
6 Cf. [3, p. 770].
} 


\section{BIBLIOGRAPHY} 520.

1. A. A. Albert, Quasigroups. I, Trans. Amer. Math. Soc. vol. 54 (1943) pp. 507-

2. R. Baer, The homomorphism theorems for loops, Amer. J. Math. vol. 67 (1945) pp. $450-460$.

3. R. H. Bruck, Simple quasigroups, Bull. Amer. Math. Soc. vol. 50 (1944) pp. 769-781.

4. - Some results in the theory of linear non-associative algebras, Trans. Amer. Math. Soc. vol. 56 (1944) pp. 141-199.

5. G. H. Garrison, Quasigroups, Ann. of Math. vol. 41 (1940) pp. 474-487.

6. F. Kiokemeister, $A$ theory of normality for quasigroups, Amer. J. Math. vol. 70 (1948) pp. 99-106.

7. B. L. van der Waerden, Moderne Algebra, Berlin, 1930, 1st ed.

MT. Holyoke College

\section{A CONJECTURE OF KRISHNASWAMI}

\section{H. LEHMER}

Let $T(N)$ denote the number of right triangles whose perimeters do not exceed $2 N$, and whose sides are relatively prime integers. A list of all such triangles whose perimeters do not exceed 10000 has been given by A. A. Krishnaswami. ${ }^{1}$ On the basis of this table he conjectured that

$$
T(N) \sim N / 7 .
$$

The asymptotic formula

$$
T(N) \sim \pi^{-2} N \log 4
$$

follows from the general theory of "totient points," as developed by D. N. Lehmer in 1900. A statement equivalent to (2) will be found in his $\operatorname{paper}^{2}$ (p. 328).

The conjecture (1) is not far wrong since

$$
\pi^{2} / \log 4=7.11941466 \text {. }
$$

Presented to the Society, April 17, 1948; received by the editors January 29, 1948.

${ }^{1}$ A. A. Krishnaswami, On isoperimetrical Pythagorean triangles, Tôhoku Math. J. vol. 27 (1926) pp. 332-348. Two omissions in Table I may be noted: For $s=3450$, $a=50, b=19$; for $s=3465, a=55, b=8$. This table is the basis for the one at the end of the present paper.

${ }^{2}$ D. N. Lehmer, Asymptotic evaluation of certain totient sums, Amer. J. Math. vol. 22 (1900) pp. 293-335. 